\title{
conference abstracts
}

s6.m2.p3 Influence of temperature and pressure on anion ordering in the crystal structures of low dimensional organic conductors. D. Chasseau, D. Le Pevelen, Y. Barrans, L. Ducasse ${ }^{1}$, J.M. Fabre ${ }^{2}$, P. Guionneau, J. Gaultier. Institut de Chimie de la Matière Condensée de Bordeaux, CNRS UPR 9048, 87 Av. Dr A. Schweitzer, 33608 Pessac Cedex, France. ${ }^{1}$. Laboratoire de Physico-Chimie Moléculaire, UMR 5803, Université Bordeaux I, 33405 Talence, France. ${ }^{2}$ Laboratoire de Chimie Organique Structurale, Université Montpellier II, Place E. Bataillon, 34095 Montpellier France.

Keywords: extreme conditions, organic conductors .

The complexity of the physical behaviour of the low dimensional organic conductors requires the investigation of the low temperature and the high pressure structural properties. These kind of results may now be obtained within the ranges $(300-7 \mathrm{~K})$ and $(0-20 \mathrm{kbar})$ by X-ray diffraction using the very low temperature device as well as the diamond anvil pressure cell developed in Bordeaux. The results of the investigation under constraints ( $\mathrm{P}$ and $\mathrm{T}$ ) we performed on $(\mathrm{TMTCF})_{2} \mathrm{X}$ and $(\mathrm{TSeT})_{2} \mathrm{Cl}$ salts will be presented.

In the series of $(\mathrm{TMTCF})_{2} \mathrm{X}$ salts $(\mathrm{C}=\mathrm{S}$ or $\mathrm{Se})$ the low temperature crystal structures have been determined in the (a, 2b, 2c) cell for the linear anion $\mathrm{X}=\mathrm{SCN}$, in the $(2 \mathrm{a}, \mathrm{b}$, c) cell for the planar anion $\mathrm{X}=\mathrm{NO}_{3}$ and in the (a, 2b, c) cell for the tetrahedral anion $\mathrm{X}=\mathrm{ClO}_{4}$. For the latter, it has been shown that the ordering phase does not appear under pressure. For (TSeT) $)_{2} \mathrm{Cl}$, a drastic change of the position of the chloride anion occurs under pressure. In all cases, the effect of the ordering on the cationic stacks has been appreciated through transfer integrals calculations. s6.m2.p4 X-ray Diffraction and Theoretical Studies of the High-Pressure Behavior of Magnesium Fluoride. J. Haines [1], J.M. Léger [1], F. Gorelli, [2], D.D. Klug [3], J.S. Tse [3] Z.Q. Li, [1] Laboratoire de Physico-Chimie des Matériaux, CNRS, 1, place Aristide Briand 92195 Meudon Cedex France, [2] LENS and INFM, Largo Enrico Fermi 2, 50125 Florence, Italy [3] Steacie Institute for Molecular Sciences, National Research Council of Canada, Ottawa, Ontario, Canada K1A 0R6.

Keywords: high pressure, phase transitions, $\mathrm{MgF}_{2}$.

Magnesium fluoride is an archetypal simple ionic solid and as such has been subject to numerous theoretical studies with particular emphasis on the rutile to fluorite phase transition. In the present study by angle-dispersive, $\mathrm{x}$-ray powder diffraction and density functional planewave methods, it is shown that the high-pressure behavior of $\mathrm{MgF}_{2}$ is much more complex. A second-order tetragonal rutile-type to orthorhombic $\mathrm{CaCl}_{2}$-type phase transition is observed prior to the transformation to the cubic phase, which is found to have a disorted fluorite structure. The structures of these three phases were refined by the Rietveld method and the pressure dependence of the structural parameters are compared with theory. 